\title{
A Relevância das Novas Tecnologias na Comunicação Organizacional: o Caso dos Websites nas Universidades Portuguesas
}

\author{
Sónia Silva, Teresa Ruão \& Gisela Gonçalves \\ Universidade da Beira Interior e Universidade do Minho / Universidade do Minho / \\ Universidade da Beira Interior \\ E-mail: sonia.c.melo.silva@gmail.com/truaodics.uminho.pt / \\ gisela.goncalves@labcom.ubi.pt
}

\section{RESUMO}

A revolução digital protagonizada pelo aparecimento da internet motivou um conjunto de transformações económicas, politicas, culturais e sociais, que provocaram profundas alterações no contexto em que se desenvolvem todas as atividades humanas. A sociedade de informação, fruto do advento desta nova tecnologia, representou um momento de importantes e decisivos progressos quer na comunicação individual quer na comunicação organizacional. É precisamente no contexto organizacional que este estudo pretende focar-se, ao discutir as potencialidades que as novas tecnologias trazem para a comunicação organizacional, tendo como particular enfoque a relevância dos websites como meios de comunicação e, as instituições de ensino superior portuguesas como estudo de caso. Para compreender se, de facto, as páginas de internet carecem de particular importância no panorama comunicativo das universidades portuguesas, utilizaram-se

Data de submissão: 09-05-2016. Data de aprovação: 23-10-2016.

Publicação realizada no âmbito da Bolsa de Doutoramento com a referência PD/BD/114002/ 2015 co-financiada pelo Fundo Social Europeu (FSE), pelo Programa Operacional Potencial Humano (POPH) e por fundos nacionais do Ministério da Educação e Ciência (MEC), através da FCT - Fundação para a Ciência e Tecnologia.

A Revista Estudos em Comunicação é financiada por Fundos FEDER através do Programa Operacional Factores de Competitividade - COMPETE e por Fundos Nacionais através da FCT - Fundação para a Ciência e a Tecnologia no âmbito do projeto Comunicação, Filosofia e Humanidades (LabCom.IFP) UID/CCI/00661/2013. 
como métodos a análise documental e a recolha de dados. Os resultados desta investigação sugerem a relevância das páginas web no contexto comunicacional

Palavras-chave: internet; websites; comunicação organizacional; universidades. das universidades portuguesas, como veículos de informação de primeira instância.

\section{RESUMO}

The digital revolution led by the rise of the internet led to a set of economic, political, cultural and social transformations which caused profound changes in the context in which all human activities are developed. The information society, the result of the emergence of this new technology, consisted of a moment of important and decisive progress in both individual and organizational communication. It is precisely the organizational context this study intends to focus on, by discussing the potential that new technologies bring to organizational com- munication, with particular focus on the relevance of websites as means of communication and Portuguese higher education institutions as a case study. To understand if, in fact, internet pages are in need of particular importance in the communicative scene of Portuguese universities, document analysis and data collection were the methods that have been used. This investigation's results suggest the relevance of web pages in the Portuguese universities communicational context, as first instance information carriers.

Palavras-chave: internet; sites; comunicação organizacional; universities.

\section{INTRODUÇÃO}

"A sociedade em que vivemos é caracterizada por um desenvolvimento tecnológico contínuo que investe no sector informático e, por consequência, nos meios de comunicação, o que modifica o estilo de vida das pessoas, as interacções sociais e as estruturas presentes (...)".

Cardoso, 2008, p. 181

O trabalho de investigação que se propõe apesentar, em seguida, trata o tema da relevância das novas tecnologias para a comunicação organizacional. 
As profundas alterações que o aparecimento da internet protagonizou em todas as esferas da sociedade e que parecem não ter fim suscitou o interesse de compreender as alterações e oportunidades que esta tecnologia trouxe para o sistema de comunicação das organizações.

Tendo-se noção de que seria muito ambicioso, tratar, nesta investigação, todas as vertentes sobre o impacto da internet na comunicação organizacional, pretende-se abordar, apenas, uma pequena parte das possibilidades trazidas por esta tecnologia. Assim, focar-se-á este estudo na relevância dos websites como meios de comunicação das organizações, utilizando como estudo de caso a importância das páginas web num tipo de organização específico, nomeadamente as universidades portuguesas.

Tiveram-se como ponto de partida para o estudo de caso as instituições de ensino superior portuguesas, pois, a experiência de investigação tem seguido o interesse em estudar o desenvolvimento da comunicação organizacional nestas instituições. Por outro lado, este tema é interessante, pois, não existem muitos estudos específicos que discutam a importância dos websites na comunicação organizacional, particularmente no contexto comunicacional das instituições de ensino superior.

Assim, a pergunta que orientará o presente trabalho é: porque é que os websites devem ser um meio de comunicação privilegiado no âmbito da comunicação organizacional, em particular nas universidades? Para responder à questão traçaram-se um conjunto de objetivos, que são eles: contextualizar e compreender o aparecimento da internet; analisar as transformações ocorridas na sociedade, em particular no seu modo de comunicar, fruto da aparição desta tecnologia; perceber o grau de penetração da internet em Portugal e o tipo de utilização que tem sido feita da mesma; examinar o impacto e as potencialidades da internet no contexto comunicacional das organizações; discutir a importância dos websites como meios de comunicação nas empresas e instituições; e debater a relevância destas páginas no seio da comunicação praticada pelas universidades portuguesas.

A metodologia utilizada neste estudo caracterizou-se pelo seu carácter qualitativo. Incluiu a análise documental de relatórios acerca da apropriação da internet pela população portuguesa, tendo-se estudado os que foram produzidos pelo OberCom - Observatório da Comunicação - nos últimos dez anos, nomeadamente: a Internet em Portugal (2003-2007); a Sociedade em Rede - A Internet em Portugal 2012; a Internet em Portugal - Sociedade em 
Rede 2014; e a Internet e o consumo de notícias online em Portugal 2015. Realizou-se, ainda, uma recolha de dados que pretendeu verificar se todas as instituições de ensino superior portuguesas (públicas, privadas e politécnicas) possuíam um website próprio. Esta recolha foi realizada com a consulta do portal da Direção Geral de Ensino Superior, onde é possível consultar a lista de todas as instituições de ensino superior portuguesas, sejam elas públicas, privadas ou politécnicas. Assim, verificou-se se as 148 instituições de ensino listadas apresentavam websites.

O presente trabalho trata-se de um estudo exploratório e organiza-se da seguinte maneira: na primeira parte abordar-se-á a história do aparecimento da internet e as transformações que trouxe para a sociedade; na segunda secção será apresentado e discutido o grau de penetração desta tecnologia em Portugal e analisado o uso que lhe é dado pelos internautas; em seguida discutirse-ão as transformações que a internet trouxe para a comunicação das organizações; na quinta parte debater-se-á pertinência dos websites como meios de comunicação para empresas e instituições; e, por fim, a última secção abordará a relevância dos websites como meios de comunicação privilegiados nas universidades portuguesas.

Com este estudo não se pretende a formulação de hipóteses rígidas, deseja-se, antes, apresentar uma discussão que possa representar uma nova e valiosa contribuição para o campo de estudo da comunicação organizacional.

\section{ReVoluÇão Digital: O AdVento da Internet}

"A criação e desenvolvimento da Internet é uma extraordinária aventura humana."

Castells, 2007, p. 25

As origens da internet são apontadas para o período da Guerra Fria, nos longínquos anos 50 e 60 do século passado. O seu aparecimento ficou a deverse a uma falha encontrada no sistema de telecomunicações dos Estados Unidos da América, que poderia comprometer o sucesso das operações militares. Foi neste contexto que os EUA procuraram desenvolver um novo sistema de comunicação.

A ARPANET surgiu neste período, como uma tecnologia de distribuição da informação em rede, criada pela Advanced Research Projects Agency (ARPA), uma agência de projetos de investigação fundada pelo Departamento 
de Defesa norte-americano. Foi desenvolvida como um sistema de comunicação militar capaz de resistir à incursão nuclear, cujo principal objetivo era interligar as bases militares e os departamentos do governo americano em rede, em tempo de guerra, bem como, “(...) alcançar a superioridade tecnológica militar sobre a União Soviética (...)” (Castells, 2007, p.26). A APARNET representou, então, a primeira manifestação e o ponto de partida para aquilo que hoje se chama de internet. Esta tecnologia atraiu o interesse da comunidade académica e científica que, ao longo de duas décadas, foi responsável pelos avanços a que se foi assistindo.

Assim, e após um período de desenvolvimentos tecnológicos, a internet tal como é atualmente conhecida surgiu nos anos 90 pelas mãos de Tim Berners - Lee, criador da World Wide Web, que possibilitou a ligação em rede entre os computadores do mundo inteiro. Berners - Lee “(...) definiu e elaborou o software que permitia tirar e introduzir informação em qualquer computador ligado através da internet (...)" (Castells, 2007, p.32).

Parece, então, que a internet teve a sua origem no contexto da investigação militar alicerçada pelo desenvolvimento científico. Apesar de a sua história ser, já, longa, o aparecimento desta tecnologia no seio das organizações e da sociedade em geral não acompanha esta longevidade:

Apesar de a Internet estar já na mente dos informáticos desde princípios dos anos 60, de em 1969 se ter estabelecido uma rede de comunicações entre computadores e, desde final dos anos 70, se terem formado várias comunidades interactivas de cientistas e hackers, para as pessoas, as empresas e para a sociedade em geral, a Internet nasceu em 1995. (Castells, 2007, p. 33)

Foi a partir deste momento que a internet ganhou expressão como meio de comunicação e, desde então, esta tecnologia tem sido protagonista de uma evolução sem precedentes. Em Portugal, tal como na generalidade dos países, a internet apareceu na década de 80. Contudo, neste período, existia apenas acesso remoto por terminal a computadores de universidades estrangeiras. Este acesso era quase exclusivamente utilizado por ex-estudantes dessas instituições de ensino superior, que mantinham as suas contas nesses sistemas (Evolução da Informática TIC - Moimenta da Beira). Porém, a ligação à internet, tal como hoje se conhece, em Portugal, também aconteceu nos anos 90, no seio universitário. A Universidade de Lisboa foi a primeira instituição 
a ter ligação a esta rede, seguindo-se, a ela, a Universidade do Minho no ano de 1993 (Origens da Internet, 2011). A partir de meados dos anos 90, quer em Portugal, quer no resto do mundo, esta nova tecnologia iniciou uma evolução sem precedentes, a que ainda se assiste nos dias de hoje. "De 1993 até à atualidade a web não tem parado de crescer, um estudo da Science, de Junho de 2011, indicava que existiam mais de 340 milhões de sites" (Carrera, 2012, p. 20).

Mas o desenvolvimento da internet não deve apenas classificar-se pelo número de aplicações e de possibilidades de utilização que foram surgindo ao longo destes 20 anos. Tal como afirma Caraça:

(...) O conjunto de mudanças experimentadas a todos os níveis, do económico ao político, do social ao cultural - e a que se foi chamando de «globalização» por uns, de «sociedade de informação» por outros, de «novo paradigma de comunicação» por outros ainda - foi de tal maneira poderoso que provocou uma alteração do contexto em que se desenvolveram as atividades humanas. (2005, p.7)

De facto, esta tecnologia de informação e comunicação deve ser pensada como palco de uma nova forma de vida em sociedade: a sociedade em rede na era da informação. Poderia falar-se apenas de sociedade em rede, porém, tal como declara Castells "as redes são formas muito antigas de actividade humana, mas actualmente estas redes ganharam uma vida nova, ao converterem-se em redes de informação, impulsionadas pela Internet" (2007, p. 15). Na verdade, a internet foi a grande impulsionadora da sociedade de informação que hoje se conhece e que impôs um conjunto de reajustamentos nos comportamentos, para que se tornasse possível beneficiar dos progressos resultantes da alteração do paradigma informacional, cujo protagonismo deixou de ser analógico para passar a ser digital. Esta nova tecnologia possibilitou desenvolvimentos antes inimagináveis, permitindo, pela primeira vez, a comunicação de muitos para muitos à escala global (Castells, 2007), abolindo as fronteiras do tempo e do espaço. A sociedade em rede na qual todos habitam é um resultado do desenvolvimento da internet.

Contudo, ainda que as novas tecnologias de informação e comunicação sejam abordadas como as principais responsáveis pela criação desta nova forma de sociedade, é de extrema relevância não descurar que o seu surgimento teve, 
por trás, um contexto histórico e cultural específico que, determinou a sua evolução. (idem). Pode, então, dizer-se que a internet transformou a sociedade no seu todo, todavia, é ela própria um produto resultante das evoluções históricas, científicas, culturais e sociais tendo surgido como um meio para suprir determinadas necessidades no seio da humanidade. Deve, portanto, ter-se em conta a existência de uma interação entre as tecnologias da informação e a sociedade, na qual ambas se influenciam mutuamente, aceitando, desta forma, que o desenvolvimento tecnológico seja algo que tenha efeitos sociais relevantes, mas que, ao mesmo tempo, é também o resultado de uma moldagem social onde interferem as características políticas, económicas e sociais do contexto onde se insere (Nunes, 2004). Não se pode, portanto, ignorar a relação de mútua influência que existe entre a internet e o meio social onde esta se insere. Um sistema mediático não se caracteriza apenas pelas tecnologias que o suportam, mas é também a própria cultura que instiga essas mudanças (Jenkins, Green \& Ford, 2014). Tal tese e corroborada por Castells que assegura que:

(...) As pessoas, as instituições, as empresas e a sociedade em geral, transformam a tecnologia, qualquer tecnologia, apropriandoa, modificando-a e experimentando-a - espacialmente no caso da Internet, por ser uma tecnologia de comunicação. (...) Como a actividade humana está baseada na comunicação e a Internet transforma o modo como comunicamos, as nossas vidas vêm-se profundamente afectadas por esta nova tecnologia de comunicação. Por outro lado, ao utilizarmos a internet para múltiplas tarefas vamos transformando-a. (2007, p. 19)

A internet deverá, então, ser continuamente pensada como um instrumento que, ao mesmo tempo, produz e é produzido por novos comportamentos da sociedade.

Contudo, esta asserção não torna menos verdade o facto de que "no one today disputes that the Internet is likely to have a significant impact on social life" (Bargh \& McKenna, 2003, p. 575). Vários autores têm vindo a estudar o impacto da internet nas várias esferas da sociedade e, parecem não restar dúvidas de que "o nosso mundo e as nossas vidas estão a experimentar uma mudança profunda no âmbito da tecnologia, economia, cultura, comunicação, política e da relação entre pessoas" (Cardoso et al, 2005, p. 13). 
Apesar do desenvolvimento da internet não ter sido propriamente veloz, a sua incursão nos modos de vida da sociedade contemporânea foi célere e discreta. Pode, até, afirmar-se que, não se sabendo muito bem como é que ela entrou na experiência quotidiana, percebe-se que é já impossível imaginá-la sem a influência desta tecnologia.

"Com a chegada da Internet, o paradigma de comunicação alterou-se por completo" (Póvoas, 2009, p. 141) e, atualmente, esta tecnologia já não é apenas um instrumento de partilha e acesso à informação para o qual foi criada. Esta primeira dimensão está ultrapassada e a internet é, agora "uma tecnologia social, onde milhares e milhões de diversos atores e sujeitos sociais interagem, criando, portanto, dimensões novas de relação social e projetando, até, porventura, novas formas de organização social" (Comunicarte, 2010, p. 14).

Como já referido, neste estudo, pretende-se dar enfoque a um tipo específico de atores sociais, que são eles as organizações. É um dos objetivos compreender o papel da internet nas empresas e instituições, em particular no que diz respeito à alteração dos seus padrões comunicativos, em consequência da adoção desta nova tecnologia, utilizando, como exemplo, a adoção dos websites pelas universidades portuguesas. Esta é uma análise interessante, pois, "actualmente, as principais actividades económicas, sociais, políticas e culturais de todo o planeta estão a estruturar-se através da Internet (...)" (Castells, 2007, p. 17) e as organizações têm vindo a estruturar as suas atividades de comunicação de acordo com as potencialidades desta tecnologia.

\section{A Internet e os seus Utilizadores em Portugal}

"A Internet, enquanto agente aglomerador de informação, é a mais intensa experiência comunicacional que a sociedade humana já viveu."

Cardoso, Mendonça, Lima, Paisana \& Neves, 2014, p. 4

Antes de se aprofundar a questão da utilização da internet como um novo paradigma para a comunicação organizacional é importante fazer-se uma pequena abordagem ao uso que os indivíduos fazem desta tecnologia, em particular a população portuguesa (uma vez que o presente estudo visa refletir sobre a importância dos websites nas universidades em Portugal). A justificação para este facto é simples: o investimento pela comunicação online por 
parte de uma organização justifica-se, apenas se o seu público-alvo estiver familiarizado com a mesma plataforma e a incluir nas suas práticas quotidianas.

Uma leitura atenta pelas conclusões dos relatórios anuais do uso da internet em Portugal (neste caso analisaram-se os que foram produzidos pelo OberCom - Observatório da Comunicação, nomeadamente: a Internet em Portugal (2003-2007), a Sociedade em Rede - A Internet em Portugal 2012, a Internet em Portugal - Sociedade em Rede 2014 e a Internet e o consumo de notícias online em Portugal 2015) permitem concluir que a utilização destas tecnologia registou grandes evoluções ao longo da primeira década do século XXI, e que, atualmente, o recurso à internet se apresenta como uma prática disseminada entre a generalidade da população portuguesa. "Cada vez mais os portugueses se relacionam com os restantes meios de comunicação e com outros formatos de visualização através da Internet" (Cardoso, Espanha, Lima \& Paisana 2012, p. 4).

Porém, a leitura dos quatro relatórios chama a atenção para a existência de uma fração de indivíduos que apresenta taxas de não utilização da internet, nomeadamente pessoas mais velhas e com menos escolaridade. Denota-se um decréscimo na utilização desta plataforma à medida que a idade aumenta e o nível de escolaridade diminui (Cardoso et al, 2012), mas, é fundamental ter em conta que nem todos têm a mesma oportunidade de acesso às tecnologias ou, as habilidades necessárias para as utilizar (Jenkins, et al, 2014). Por outro lado, são os indivíduos na faixa etária entre os 15 e os 24 anos, que apresentam também maior grau de formação académica, os maiores utentes desta tecnologia (a taxa de utilização neste grupo é 94,1\%) (Cardoso et al, 2014). Contudo, neste momento,

(...) A utilização da internet tende cada vez mais a ultrapassar as barreiras demográficas, ditas «tradicionais», que se impõe na utilização de novas tecnologias. (...) Cada vez mais surgem recursos que procuram atrair novos públicos, mesmo que pouco literados em termos tecnológicos". (Cardoso, Mendonça, Lima, Paisana \& Neves, 2014, p. 8)

Embora ainda se registe a presença de grupos omissos na utilização desta plataforma, é também um facto que a maioria dos portugueses a utiliza diariamente, tendo vindo a registar-se um aumento dos acessos à rede nos últimos anos (Cardoso et al, 2014). Pode mesmo afirmar-se que recorrer aos serviços 
da internet se tornou num ato tão simples como utilizar o telefone (Marcelo, 2005). Hoje em dia "o ciberespaço é o lugar (...) onde as pessoas acessam, recupera, organizam, ensinam, disseminam e compartilham informação e conhecimento" (Luvizotto, Fusco \& Scanavacca, 2010, p. 26).

No que respeita aos usos atribuídos à internet, estes são vários. Estabelecer comunicação com outrem, procurar atividades de entretenimento, aceder aos conteúdos dos média tradicionais e procurar informação sobre diversos bens e serviços são as ações mais procuradas pelos internautas portugueses. Na verdade, os utilizadores da internet “(...) são pessoas comuns que se ligam à rede com o intuito de desenvolverem com mais facilidade as suas tarefas do dia a dia, como seja, por exemplo, comunicar ou procurar informações (...)" (Marcelo, 2005, p. 275). Pode declarar-se, então, que a internet é, principalmente, um meio de consulta de informação, a que os indivíduos recorrem para saber mais sobre determinados produto e serviços, facilitando a sua escolha (Cardoso et al, 2012). Nesta função de procura de informação tem sido atribuído algum destaque aos websites. "A segunda fonte online com mais importância atribuída são os sites em geral (...). Os sites oficiais de instituições públicas também obtêm avaliação positiva (...)” (Cardoso et al, 2014).

Tendo em conta o contexto acima descrito parece que as organizações encontram um contexto favorável para o desenvolvimento da sua comunicação online, em particular dos seus websites. Pode até dizer-se que a sua presença no mundo online é essencial para chegar aos seus públicos. Quando se fala de instituições de ensino superior este facto assume especial relevância, pois, como se constatou, os indivíduos entre os 15 e os 24 anos são os que mais recorrem à internet para as suas atividades do quotidiano e, com certeza, utilizá-la-ão para procurar informações sobre as universidades onde pretendem ingressar ou sobre a instituição que já se encontram a frequentar.

Assim, daqui em diante será relevante analisar a internet como um paradigma recente para a comunicação organizacional, em especial, para as instituições de ensino superior em Portugal.

\section{A Internet como um Paradigma para a Comunicação Orga- NIZACIONAL}

"Numa sociedade onde as empresas (...) são a principal fonte de criação de riqueza, não deveria surpreender-nos que, assim que 
a tecnologia da Internet ficou disponível, nos anos 90, a difusão mais rápida e ampla das suas utilizações tivesse tido lugar no âmbito da empresa. A Internet está a transformar a prática empresarial (...). O uso apropriado da Internet converteu-se numa fonte fundamental de produtividade e competividade para todo o tipo de empresas."

Castells, 2007, p. 87

O termo comunicação organizacional apresenta uma vastidão de definições. Para a presente investigação ter-se-á em conta o sentido proposto por Mumby, 2001, que expõe a comunicação organizacional como "o processo de criação de estruturas de significado, colectivas e coordenadas, através de práticas simbólicas orientadas para atingir objectivos organizacionais" (citado em Ruão, 2008, p. 27). Comunicação organizacional é, pois, a criação e gestão de representações e sistemas simbólicos capazes de gerar efeitos em públicos específicos (Poupinha \& Espanha, 2005).

Atualmente os termos comunicação organizacional, comunicação empresarial e comunicação institucional são reconhecidos e estão bastante difundidos, pois, também as organizações dão cada vez mais atenção às suas práticas comunicacionais. Não quer isto dizer que a existência de atividades de comunicação nas empresas e instituições seja algo relativamente recente. $\mathrm{Na}$ verdade, a comunicação está no âmago da existência de qualquer organização, tal como afirma Ruão:

(...) A comunicação é anterior ao estabelecimento de uma organização e que constitui uma dimensão que acompanha toda a sua vida, porque as organizações necessitam permanentemente de partilhar informações, ideias ou pensamentos. Aliás, a comunicação parece estar na base de todas actividades de cooperação entre os seres humanos, que é a noção que preside às organizações". (2008, p. 27)

Contudo, por vezes, a noção de comunicação organizacional aparece estritamente ligada à gestão de ações de comunicação por parte das organizações, destinadas a promover a sua atividade quando, na verdade, se trata de toda a interação que as organizações desenvolvem com o ambiente que as rodeia (Ruão, 2001). É possível declarar que a comunicação nas organizações não é algo isolado ou separado da sua natureza e, portanto, pode ser visto como 
algo estritamente ligado à sua existência. A comunicação é crucial na vida humana e nas organizações, porque ela constitui o processo central de organizar. É a comunicação que permite às pessoas gerar e partilhar informações, e por, neste sentido, que lhes permite constituírem-se através de organizações (Ruão, Salgado, Freitas e Ribeiro, 2014).

No entanto, só mais tarde as organizações começaram a dar atenção as suas atividades comunicativas que são, atualmente, uma prática disseminada no cerne da sua atuação diária. Tal como defende Ruão:

(...) Ao longo da década de 1990, assistimos à multiplicação das actividades organizacionais destinadas a promover a visibilidade e o reconhecimento público das empresas, através de programas de comunicação que geriam as impressões, os activos intangíveis, a competência simbólica ou a dimensão expressiva das organizações". (2008, p. 12)

O aparecimento da internet, nos anos 90 veio revolucionar a atividade das organizações. O surgimento desta tecnologia representou um fator central da nova forma de organização social, que trouxe alterações substanciais nas práticas comunicacionais (Cardoso et al, 2005). As empresas e instituições descobriram um instrumento privilegiado para comunicar com os seus públicos pois, a internet trouxe múltiplas possibilidades de contacto. As organizações tiveram que se reestruturar em função desta nova tecnologia, que veio alterar as suas formas de relacionamento. (Castells, 2007).

Tal como aconteceu com os indivíduos, a penetração da internet no seio das organizações foi acontecendo de forma gradual. Mas, nos dias de hoje é impensável imaginar uma empresa ou instituição fora desta plataforma, que é já uma extensão da própria organização, sem a qual a mesma põe em risco a sua existência. "De facto, a exclusão destas redes é uma das formas de exclusão mais graves que se pode sofrer na nossa economia e na nossa cultura" (Castells, 2007, p. 17).

Atualmente, é raro encontrar uma empresa ou instituição que não possua um website e uma página em, pelo menos, numa rede social, sendo que, a maioria prefere difundir a sua atividade em várias redes, nomeadamente $o$ Facebook, o Twitter, o Instragram, o Linkedin, e em muitas outras que vão surgindo diariamente. "As redes são e serão os componentes fundamentais das organizações” (Castells, 2001, p. 188). Estamos perante um momento 
em que a utilização da internet se expandiu a todo o tipo de organizações, em operações de diversas índoles. Pode-se, portanto, concluir que se está perante um novo mundo organizacional (Castells, 2007), onde as empresas e instituições ganham autonomia para comunicar com os seus públicos (Póvoas, 2009).

Este contexto torna-se ainda mais relevante quando as organizações percebem que os seus públicos também estão na internet. Assim, “(...) dão-lhe agora mais atenção do que nunca - desejam marcar presença, porque reconhecem que os seus públicos estão «ligados» à rede (...)” (Comunicarte, 2010, p. 14).

Parece, pois, que a internet se constitui como um mundo privilegiado de oportunidades, onde as organizações podem explorar novas formas de negócio, promovendo, ao mesmo tempo, um contacto mais pessoal, direcionado e interativo com os seus públicos (Marques, 2012). Hoje em dia, em qualquer organização “(...) a web é uma ferramenta essencial para a divulgação, promoção e comercialização de produtos/ serviços; para a interação com o consumidor final; para o desenvolvimento de estratégias de comunicação, marketing e publicidade (...)" (Comunicarte, 2010, p. 91) e, por isso, o espaço online tornou-se uma ferramenta extremamente relevante nas suas práticas comunicacionais, um meio de a privilegiar no âmbito da comunicação institucional (Suarez, Moreira \& Carrapatoso, 2006).

Todavia, a internet constitui-se, também, como um grande desafio para as empresas e instituições. Ela não para de evoluir, é um planeta em constante mutação, "o futuro acontece todos os dias, está ao virar da porta. As tecnologias web encontram terreno fértil para surgirem e para se desenvolverem" (Comunicarte, 2010, p. 222). Esta é uma área sem certezas, que se caracteriza pela evolução constante, onde a organizações, para obterem o pretendido sucesso, necessitam de estar na linha da frente, estando sempre a par das novas tendências e acompanhando as necessidades e as pisadas que os seus públicos vão dando neste meio, múltiplo de oportunidades.

Para esta investigação, sabendo que é impossível debater todas as potencialidades trazida pela internet, pretende-se dar um pequeno contributo para a discussão acerca da importância dos websites como elementos presente nas práticas de comunicação das organizações, pois acredita-se que têm uma pertinência particular. Tratar-se-á, de seguida, essa questão. 


\section{OS Websites Como um Meio de CoMUniCAÇÃo POR EXCELÊNCIA}

“(...) Quando os websites corporativos surgiram, em meados dos anos 1990, ninguém percebeu por inteiro como eles iriam mudar substancialmente a relação da empresa com o seu público. Poucas empresas, que nessa época criaram websites parecendo brochuras, consideravam completamente que as marcas tinham ali a oportunidade de contar sua história diretamente para um público (...)".

Jenkins, Green \& Ford, 2014, p. 49

Não se pretende, de forma alguma, reduzir a crescente relevância de outros meios de comunicação que surgiram com a internet, como as redes sociais ou os blogs. Deseja-se, apenas, mostrar que, em determinados tipos de organizações, os websites, pelas características que possuem, poderão apesentar-se como plataformas bastante importantes para a comunicação com os seus públicos.

Nos dias que correm é um desafio encontrar organizações que não tenham uma página web (Carrera, 2012). Com os websites, “(...) as organizações têm agora um espaço próprio de definição do seu discurso e do seu universo, podendo posicionar-se tanto esteticamente quanto a nível do conteúdo" (Poupinha e Espanha, 2005, p. 214). Estas páginas na internet tornam-se um bom meio de comunicação, permitindo uma comunicação mais eficaz e atrativa, que inclui conteúdos como o vídeo, a imagem, o som e, claro, o texto. Por outro lado, este meio de comunicação oferece mais liberdade aos utilizadores. Os públicos interessados podem permanecer o tempo que desejarem em cada website, e podem ser eles próprios a definir o seu padrão de consulta de informação, navegando livremente entre páginas (Marques, 2012).

É verdade que nem sempre foi assim, os primeiros websites eram bastante simplistas e pouco atrativos, privilegiando-se o texto como veículo de informação, o que resultava em páginas entediantes e desinteressantes do ponto de vista estético. Porém, tal como a própria internet, também os websites têm sido alvo de profundos progressos. Atualmente o texto deu lugar à imagem $\mathrm{e}$ ao vídeo e as possibilidades em termos de conteúdos são muito mais diversas. A sua constante atualização, quer em termos de informação, quer em termos de layout são uma preocupação contínua das empresas e instituições, pois, os websites também representam, cada vez mais, importantes fatores de concor- 
rência. Não há dúvida que, “(...) o consumidor espera uma boa experiência no âmbito da Web (...) os utilizadores da internet mudam para um site concorrente quando o site que estão a ver tem uma má performance" (Marques, 2012, p. 36). Assim, para adquirirem um lugar de destaque, as organizações devem ter o cuidado de preparar e colocar, frequentemente, novos conteúdos nas suas páginas sendo capazes de renovar, periodicamente, as informações que sobre si circulam na internet (Póvoas, 2009).

Mas, atualmente, a tónica não é apenas colocada na atualização de informações. Os consumidores procuram mais possibilidades. Buscam conteúdos mais interativos e dinâmicos. Um bom website deverá conter diversos formatos informativos, assim, deverá incluir notícias, informações úteis, hiperligações, elementos áudio, vídeo, imagem, texto e possibilidade de alguma interação com a organização (Marques, 2012). Tal aspeto é referido por Poupinha et al:

A actualização das informações dos sites tem que ser rápida (...) sob pena de não responder à demanda daqueles que lá procuram informação (...). Uma vez fornecida a informação necessária, a ênfase é necessariamente colocada no âmbito da retórica e da estética dos sites, neste caso, ou outras formas de comunicação pela net, pelo modo como permitem, por um lado, uma relação mais agradável entre público e organização (...). (2005, p. 215).

Os websites são plenos em termos de oportunidades de comunicação e adquiriram uma importância impar na sociedade atual. É possível, até, dizer-se que têm a mesma relevância do que o contacto direto ou que o contacto telefónico com uma organização. Com isto não se quer aferir que as páginas da internet substituem estas formas tradicionais de comunicação, mas antes que poderão ser o primeiro ponto de contacto entre os públicos e as empresas e instituições, pois, geralmente incluem as informações mais importantes sobre as mesmas. "Deste modo, considera-se o sítio Web com a mesma importância, ou mais, do que outras formas de alcançar o público-alvo, deve ser construído e aperfeiçoado com pelo menos os mesmos cuidados e investimentos dispensados a outros média" (Suarez et al, 2006, p. 3).

Com as páginas web as organizações encontram um espaço próprio para a definição e difusão do seu discurso e do seu universo, podendo eleger o seu posicionamento quer em termos de conteúdo, quer em termos estéticos 
(Poupinha \& Espanha 2005) e, têm ainda a vantagem de chegar de forma mais rápida, eficaz e económica a um maior número de indivíduos. Os websites permitem que as empresas e instituições se apresentem aos seus públicos, sem intermediário.

Por outro lado, os websites têm acompanhado as constantes evoluções no mercado tecnológico, tendo-se tornado responsives, ou seja, páginas nas quais os seus elementos sejam adaptáveis aos tamanhos dos diferentes ecrãs que hoje são utilizados para aceder à internet, nomeadamente os tablets e os smartphones. Desta forma, fazem com que o seu acesso seja ainda mais isento de tempo e espaço, possibilitando aos públicos a consulta em qualquer hora e lugar.

A comunicação com os indivíduos é favorecida com este meio, pois, hoje em dia a internet é o tecido do quotidiano (Castells, 207), o que significa que grande parte das atividades diárias se fazem com o seu auxílio. Desta forma, não parece estranho que, quando procuram informação sobre algum produto ou serviço, os indivíduos procurem, numa primeira fase, obter informação através dos websites das organizações. Tal facto é compartilhado por Jenkins et al:

Ocorreria então uma mudança fundamental na maneira como todos «consomem», na maneira como as pessoas interessadas poderiam buscar conteúdo nas empresas quando quisessem - justapondo e avaliando as mensagens corporativas diretamente obtidas da fonte e publicando o que encontrassem on-line entre seus círculos de amigos, familiares, colegas e até desconhecidos. (2014, p. 49)

É com base nestes pressupostos que se atribui uma grande relevância aos websites como meios de comunicação das organizações. Claro que, a sua importância será variável consoante o tipo de empresa ou instituição que se trata. Assim, a pertinência do website poderá ser maior consoante o peso da componente informação na organização em causa. Quando se fala de instituições de ensino superior - é a este ponto que se pretende chegar - fala-se de organizações em que o elemento informativo é extremamente significante. É por isso mesmo que se acredita que, para estas instituições, o website poderá ser o seu meio de comunicação online mais importante. 


\section{Os Websites na Comunicação das Universidades Portuguesas}

"As tecnologias da Web estão redesenhando e redefinindo a transmissão (...) de conteúdos informacionais, criando novas e interessantes oportunidades de divulgação, mais personalizadas, sociais e flexíveis, com um carácter de compartilhamento de informações que vem sendo de grande valia para instituições educacionais (...)."

Luvizotto, Fusco \& Scanavacca, 2010, p. 38

O sistema universitário português é bastante jovem, remontando as suas origens para os anos 70 do século passado. A consciência da importância da comunicação organizacional nestas instituições é ainda mais recente. A preocupação com a gestão das atividades de comunicação surgiu em meados dos anos 90, quando as universidades viram a sua sobrevivência ameaçada devido à falta de financiamento por parte do Estado, à diminuição do número de alunos e à crescente concorrência entre estas instituições, fruto da proliferação de estabelecimentos de ensino públicos, privados e politécnicos. De facto, "Em menos de três décadas (...) as instituições académicas passaram de um cenário e futuro previsível e fácil recrutamento de estudantes, sem grande concorrência, para um clima de grande instabilidade" (Ruão, 2005, p. 3).

Foi, então, num contexto de crise no ensino superior que a função de comunicação se tornou mais visível nestas instituições e passou a fazer parte das suas opções estratégicas, tendo surgido, nesta altura, os primeiros gabinetes de comunicação, exclusivamente responsáveis por estas tarefas. Tal aspeto é referido por Ruão:

As instituições de ensino superior público em Portugal despertaram para a gestão controlada da comunicação e da identidade, em meados da década de 1990. (...). Nessa época, as universidades nacionais começaram a alargar os seus departamentos de comunicação, a modernizar as suas simbologias, a apostar em novos meios, o usar estratégias de segmentação e a promover activamente o trabalho científico. (2008, p. 153)

Nesta altura, as instituições de ensino superior portuguesas adotaram um novo paradigma de comunicação, mais próximo do modelo empresarial pois, precisam de desenvolver uma vantagem competitiva baseada num sistema de 
características únicas e comunicá-las, de uma maneira eficaz, a todos os públicos (Carrillo e Ruão, 2005). Para tal, estas instituições começaram a considerar novos públicos, a desenvolver novos eixos de comunicação e a apostar em novas estratégias de comunicação.

No conjunto das novas estratégias de comunicação adotadas, as novas tecnologias assumiram um papel de relevância, até porque, o incremento das atividades de comunicação nas universidades portuguesas se fez a par do surgimento da internet, em meados dos anos 90. Assim, o aparecimento das novas tecnologias marcou o início do século XXI e o período de reforma do sistema universitário português:

(...) A emergência da chamada "Sociedade do Conhecimento" (ou "da Informação"), na segunda metade do século XX, trouxera consigo extraordinárias Oportunidades (...). Referimo-nos a uma sociedade baseada na informação e dominada pela revolução tecnológica, que se tornaram eixos do desenvolvimento sustentável e da competitividade das nações. (Ruão 2008, p 157)

Acerca deste aspeto é importante ter-se como referência que, em Portugal, as universidades foram as primeiras instituições a adotar a internet, aliás, a comunidade académica foi pioneira na utilização desta tecnologia. Portanto, não é de estranhar que, cedo, tenha sido atribuída importância às potencialidades da internet como meio de comunicação.

No seio das instituições de ensino superior portuguesas a criação de websites institucionais representou uma das primeiras medidas relacionadas com o incremento das novas tecnologias. Com a proliferação de páginas de internet por parte de organizações das mais variadas áreas, as universidades perceberam que esta poderia representar uma boa oportunidade de comunicação. Por outro lado, as instituições de ensino superior de países vizinhos e, em particular, da Europa tinham, já, começado a adotar esta tendência. Na verdade, a adoção "(...) das novas tecnologias e da capacidade de inovação são traços que existiram sempre na Universidade. Eram já preocupações iniciais, mas que não se puderam concretizar logo, ou concretizaram-se à dimensão da época (...)" (Lúcio Craveiro da Silva citado em Ruão, 2008, p. 430).

Como consequência desta conjuntura, atualmente, todas as instituições de ensino superior têm o seu website (tal como se pode comprovar pela análise da tabela 1) e este é, talvez, o seu elemento de comunicação mais importante. 
“(...) The World Wide Web (WWW) and print media are perceived to be the most important sources of university information (...)" (Gray, Fam \& Llanes, 2003: 112). A recolha de dados realizada (tabela 1), permitiu constatar que, das 148 instituições de ensino superior nacionais (listadas na página web da Direção Geral de Ensino Superior), todas possuem uma página oficial. Este facto comprava a importância que este meio de comunicação possui no seio das universidades. E, é necessário referir que, neste estudo, não foram analisados os websites de cada faculdade, dentro das próprias universidades. Quer isto dizer, que, atualmente é também muito comum que as próprias faculdades possuam as suas próprias páginas web, extrapolando-se, já, a existência de apenas um website oficial da instituição de ensino superior como um todo. Pode-se justificar este contexto pela constante necessidade de informação mais específica sobre cada faculdade ou instituto.

De facto, grande parte das vezes, as páginas web das instituições de ensino superior representam o primeiro elemento de contacto com os potenciais estudantes. Não raras vezes, quando procuram informações sobre os cursos e universidades para procederem à sua candidatura ao ensino superior, os estudantes fazem-no através da internet primeiro, e só depois procuram outras fontes para se esclarecerem, como o contacto telefónico ou presencial. Este aspeto não é estranho, pois, as páginas web das instituições de ensino superior agregam informação essencial sobre seus projetos de ensino e investigação, e sobre todos os serviços de apoio aos estudantes. Desta forma, pode afirmarse que os websites são a porta de entrada para as universidades e, por isso a sua construção deve ser "(...) integrada e coerente com uma boa imagem corporativa da organização (...)” (Suarez et al, 2006, p. 3).

As instituições de ensino superior portuguesas têm demonstrado consciência da importância destes meios de comunicação. Uma pequena visita pelas páginas das universidades portuguesas permite perceber isso mesmo, denotando-se um grande investimento na frequente atualização de conteúdos e, também, na constante alteração de layouts, com vista a torná-los mais apelativos e interativos.

Porém, ainda que aqui se apresentem os websites como elementos de comunicação muito importantes para as universidades portuguesas é preciso ter em conta que estes não são os únicos. As universidades têm feito grandes desenvolvimentos nas suas atividades promocionais e, atualmente, 
Os formatos de comunicação mais usados para atingir estes públicos podem classificar-se em ações de relações públicas (que incluem as feiras e exposições, visitas a escolas, dias de portas abertas ou publicações), de publicidade (nos jornais, rádio, televisão, outdoors, cartazes ou flyers) e de merchandising (que incluem lembranças institucionais e peças de vestuário). (Ruão, 2008: 456)

Em jeito de conclusão deste capítulo pode-se referir que as universidades têm todo o interesse em apostar na qualidade dos seus websites pois, apesar de não serem um meio de comunicação recente, não deixam de ser decisivos para o contacto com os públicos. Todavia, as páginas web não devem ser tidas como o único meio de comunicação a privilegiar. Preferencialmente, estes devem ser parte de uma estratégia de comunicação que congregue outros formatos de comunicação online e offline, onde devem constar os tradicionais meios como as brochuras, os cartazes ou o merchandising, mas também, as ferramentas online mais recentes, onde as redes sociais, como fenómeno relevante de partilha e interação com os públicos, tem assumido protagonismo.

\begin{tabular}{|c|c|c|c|c|}
\hline Instituição & Pública & Politécnico & Privada & Website \\
\hline Instituto Universitário de Lisboa & $\mathrm{X}$ & & & Sim \\
\hline Universidade Aberta & $X$ & & & Sim \\
\hline Universidade dos Açores & $X$ & & & Sim \\
\hline Universidade do Algarve & $\mathrm{X}$ & & & Sim \\
\hline Universidade de Aveiro & $\mathrm{X}$ & & & Sim \\
\hline Universidade da Beira Interior & $\mathrm{X}$ & & & Sim \\
\hline Universidade de Coimbra & $\mathrm{X}$ & & & Sim \\
\hline Universidade de Évora & $\mathrm{X}$ & & & Sim \\
\hline Universidade da Madeira & $\mathrm{X}$ & & & Sim \\
\hline Universidade do Minho & $\mathrm{X}$ & & & Sim \\
\hline Universidade Nova de Lisboa & $\mathrm{X}$ & & & Sim \\
\hline Universidade do Porto & $\mathrm{X}$ & & & Sim \\
\hline Universidade Técnica de Lisboa & $\mathrm{X}$ & & & Sim \\
\hline $\begin{array}{l}\text { Universidade de Trás-os-Montes e Alto } \\
\text { Douro }\end{array}$ & $\mathrm{X}$ & & & Sim \\
\hline $\begin{array}{l}\text { Escola Superior de Enfermagem de Coim- } \\
\text { bra }\end{array}$ & & $X$ & & Sim \\
\hline
\end{tabular}


Escola Superior de Enfermagem de Lisboa Escola Superior de Enfermagem do Porto Escola Superior de Hotelaria e Turismo do Estoril

Escola Superior Náutica Infante D. Henrique

Instituto Politécnico de Beja

Instituto Politécnico de Bragança Instituto Politécnico de Castelo Branco Instituto Politécnico do Cávado e do Ave Instituto Politécnico de Coimbra Instituto Politécnico da Guarda Instituto Politécnico de Leiria

Instituto Politécnico de Lisboa

Instituto Politécnico de Portalegre

Instituto Politécnico do Porto

Instituto Politécnico de Santarém

Instituto Politécnico de Setúbal

Instituto Politécnico de Tomar

Instituto Politécnico de Viana do Castelo

Instituto Politécnico de Viseu

Escola Superior Artística do Porto

Escola Superior de Atividades Imobiliárias

Escola Superior Gallaecia

Escola Universitária das Artes de Coimbra

Escola Universitária Vasco da Gama

Instituto de Arte, Design e Empresa

Instituto Superior Bissaya Barreto

Instituto Superior D. Afonso III

Instituto Universitário de Ciências da Saúde

$\mathrm{X}$

$\mathrm{X}$

$\mathrm{X}$

X

Sim

Sim

Sim

Sim

$\mathrm{X}$

Sim

$\mathrm{X}$

Sim

$\mathrm{X}$

Sim

X

Sim

X

Sim

X

Sim

Sim

Sim

Sim

Sim

Sim

Sim

Sim

X

Sim

$\mathrm{X}$

Sim

$\mathrm{X}$

X Sim

X Sim

X Sim

X Sim

X Sim

X Sim

X Sim

X Sim

X Sim

Instituto Superior de Ciências da Saúde

X Sim

Egas Moniz

Instituto Superior de Educação e Trabalho

Instituto Superior de Estudos Interculturais

e Transdisciplinares - Almada
X Sim

X Sim 
Instituto Superior de Estudos Interculturais

e Transdisciplinares - Viseu

Instituto Superior de Gestão

Instituto Superior de Línguas e Administra-

ção de Leiria

Instituto Superior de Serviço Social do

Porto

Instituto Superior Manuel Teixeira Gomes

Instituto Superior Miguel Torga

Instituto Universitário da Maia

Instituto Universitário de Ciências Psicoló-

gicas, Sociais e da Vida

Universidade Atlântica

Universidade Autónoma de Lisboa Luís de

Camões

Universidade Católica Portuguesa - Centro

Regional das Beiras

Universidade Católica Portuguesa - Escola das Artes

Universidade Católica Portuguesa - Escola

Superior de Biotecnologia

Universidade Católica Portuguesa - Facul-

dade de Ciências Económicas e Empresari-

ais

Universidade Católica Portuguesa - Facul-

dade de Ciências Humanas

Universidade Católica Portuguesa - Facul-

dade de Ciências Sociais

Universidade Católica Portuguesa - Facul-

dade de Direito

Universidade Católica Portuguesa - Facul-

dade de Direito (Porto)

Universidade Católica Portuguesa - Facul-

dade de Economia e Gestão

Universidade Católica Portuguesa - Facul-

dade de Educação e Psicologia

\begin{tabular}{|c|c|}
\hline$X$ & Sim \\
\hline$X$ & Sim \\
\hline$X$ & Sim \\
\hline$X$ & Sim \\
\hline$X$ & Sim \\
\hline$X$ & Sim \\
\hline$X$ & Sim \\
\hline$X$ & Sim \\
\hline$X$ & Sim \\
\hline$X$ & Sim \\
\hline$X$ & Sim \\
\hline$X$ & Sim \\
\hline$X$ & Sim \\
\hline$X$ & Sim \\
\hline$X$ & Sim \\
\hline$X$ & Sim \\
\hline$X$ & Sim \\
\hline$X$ & Sim \\
\hline$X$ & Sim \\
\hline$X$ & Sim \\
\hline
\end{tabular}


Universidade Católica Portuguesa - Facul-

X Sim

dade de Filosofia

Universidade Católica Portuguesa - Facul-

dade de Teologia

Universidade Católica Portuguesa - Facul-

dade de Teologia (Braga)

Universidade Católica Portuguesa - Facul-

dade de Teologia (Porto)

Universidade Católica Portuguesa - Insti-

tuto de Bioética

Universidade Católica Portuguesa - Insti-

tuto de Ciências da Saúde

Universidade Católica Portuguesa - Insti-

tuto de Ciências da Saúde (Porto)

Universidade Católica Portuguesa - Insti-

tuto de Estudos Europeus

Universidade Católica Portuguesa - Insti-

tuto de Estudos Políticos

Universidade Europeia

Universidade Fernando Pessoa

Universidade Lusíada

Universidade Lusíada do Porto

Universidade Lusíada de Vila Nova de Fa-

malicão

Universidade Lusófona de Humanidades e

Tecnologias

Universidade Lusófona do Porto

Universidade Portucalense Infante D. Hen-

rique

Academia Nacional Superior de Orquestra

CESPU Escola Superior de Saúde de Vale

do Ave

CESPU Escola Superior de Saúde de Vale

do Sousa

Conservatório Superior de Música de Gaia

\begin{tabular}{ll} 
X & Sim \\
X & Sim \\
& \\
X & Sim \\
& \\
X & Sim \\
& \\
X & Sim \\
& \\
X & Sim \\
& \\
X & Sim \\
& \\
X & Sim \\
& \\
X & Sim \\
X & Sim \\
X & Sim \\
X & Sim \\
X & Sim \\
X & Sim \\
& \\
X & Sim \\
& \\
\hline & \\
& \\
& \\
X Sim \\
X
\end{tabular}


Escola Superior Artística do Porto (Guima-

rães)

Escola Superior de Artes Decorativas

Escola Superior de Artes e Design

Escola Superior de Educação de Almeida

Garrett

Escola Superior de Educação de Fafe

Escola Superior de Educação de João de

Deus

Escola Superior de Educação de Paula Frassinetti

Escola Superior de Educação de Torres Novas

Escola Superior de Educação Jean Piaget de

Almada

Escola Superior de Educação Jean Piaget de

Arcozelo

Escola Superior de Educação Jean Piaget de Arcozelo (Viseu)

Escola Superior de Educação Jean Piaget do Nordeste

Escola Superior de Educadores de Infância Maria Ulrich

Escola Superior de Enfermagem da Cruz Vermelha Portuguesa de Oliveira de Azeméis

Escola Superior de Enfermagem de S. José de Cluny

Escola Superior de Enfermagem de Santa Maria

Escola Superior de Enfermagem Dr. José Timóteo Mantalvão Machado

Escola Superior de Enfermagem S. Francisco das Misericórdias

Escola Superior de Saúde da Cruz Vermelha Portuguesa

\begin{tabular}{|c|c|}
\hline$X$ & Sim \\
\hline$X$ & Sim \\
\hline$X$ & Sim \\
\hline$X$ & Sim \\
\hline$X$ & Sim \\
\hline$X$ & Sim \\
\hline$X$ & $\mathrm{Sim}$ \\
\hline$X$ & Sim \\
\hline$X$ & $\mathrm{Sim}$ \\
\hline$X$ & Sim \\
\hline$X$ & $\mathrm{Sim}$ \\
\hline$X$ & $\mathrm{Sim}$ \\
\hline$X$ & $\mathrm{Sim}$ \\
\hline$X$ & $\mathrm{Sim}$ \\
\hline$X$ & Sim \\
\hline$X$ & Sim \\
\hline$X$ & Sim \\
\hline$X$ & Sim \\
\hline$X$ & Sim \\
\hline
\end{tabular}


Escola Superior de Saúde de Alcoitão

Escola Superior de Saúde Egas Moniz

X Sim

Escola Superior de Saúde Jean Piaget - Al-

X Sim

garve

Escola Superior de Saúde Jean Piaget de

X Sim

Vila Nova de Gaia

Escola Superior de Saúde Jean Piaget de Vi-

X Sim

seu

Escola Superior de Saúde Jean Piaget/ Nor-

deste

Escola Superior de Saúde Ribeiro Sanches

Escola Superior de Tecnologia e Gestão

X Sim

Jean Piaget do Litoral Alentejano

Escola Superior de Tecnologias de Fafe

Escola Superior de Tecnologias e Artes de

X Sim

Lisboa

Instituto de Estudos Superiores Financeiros

e Fiscais do Porto

Instituto Português de Administração de

X Sim

X Sim

Marketing de Lisboa

Instituto Português de Administração de

Marketing do Porto

Instituto Português de Administração de

X Sim

X Sim

Marketing de Aveiro

Instituto Superior Autónomo de Estudos

Politécnicos

Instituto Superior D. Dinis

Instituto Superior de Administração e Ges-

X Sim

tão

Instituto Superior de Administração e Lín-

X Sim

X Sim

X Sim

guas

Instituto Superior de Ciências da Adminis-

tração

Instituto Superior de Ciências da Informa-

ção e da Administração

Instituto Superior de Ciências Educativas

X Sim

X Sim

X Sim

X Sim

X Sim

X Sim

X Sim 
Instituto Superior de Ciências Educativas do Douro

Instituto Superior de Comunicação Empresarial

Instituto Superior de Educação e Ciências

Instituto Superior de Espinho

Instituto Superior de Gestão Bancária

Instituto Superior de Novas Profissões

Instituto Superior de Paços de Brandão

Instituto Superior de Saúde do Alto Ave

Instituto Superior de Tecnologias Avança-

das de Lisboa

Instituto Superior de Tecnologias Avança-

das de Lisboa (Porto)

Instituto Superior Politécnico de Oeste

Instituto Superior Politécnico Gaya - Es-

cola Superior de Ciências e Tecnologia

Instituto Superior Politécnico Gaya - Es-

cola Superior de Desenvolvimento Social e

Comunitário

Instituto Superior Politécnico Gaya - Es-

cola Superior de Educação de Santa Maria

ISLA - Instituto Politécnico de Gestão e

Tecnologia

ISLA - Instituto Superior de Gestão e Administração de Santarém

Universidade Atlântica - Escola Superior

de Saúde Atlântica

Universidade Católica Portuguesa - Escola

Superior Politécnica de Saúde (Lisboa)

Universidade Católica Portuguesa - Escola

Superior Politécnica de Saúde (Porto)

Universidade Fernando Pessoa - Escola Su-

perior de Saúde

\begin{tabular}{|c|c|}
\hline$X$ & Sim \\
\hline$X$ & Sim \\
\hline$X$ & Sim \\
\hline$X$ & Sim \\
\hline$X$ & Sim \\
\hline$X$ & Sim \\
\hline$X$ & Sim \\
\hline$X$ & Sim \\
\hline$X$ & Sim \\
\hline$X$ & Sim \\
\hline$X$ & Sim \\
\hline$X$ & Sim \\
\hline$X$ & Sim \\
\hline$X$ & Sim \\
\hline$X$ & Sim \\
\hline$X$ & Sim \\
\hline$X$ & Sim \\
\hline$X$ & Sim \\
\hline$X$ & Sim \\
\hline$X$ & Sim \\
\hline
\end{tabular}

Sim

im

m

Sim

m

iim

im

$\operatorname{Sim}$

$\operatorname{sim}$

im

m

im 
Tabela 1. Tabela de verificação da presença de websites nas 148 instituições de ensino superior portuguesas.

\section{CONCLUSÕES}

"A Internet é o tecido das nossas vidas."

Castells, 2007, p. 15

O presente estudo pretendeu compreender a relevância das novas tecnologias, em particular dos websites, para a comunicação no contexto organizacional, a partir da análise do caso das páginas web nas universidades portuguesas.

Parecem não restar dúvidas de que o aparecimento da internet trouxe consigo um conjunto de transformações que se sentiram a todos os níveis, desde o cultural, ao económico, ao político e ao social. O principal impacto desta tecnologia foi, talvez, o incremento da sociedade de informação que foi responsável por progressos decisivos na forma de comunicação das sociedades. A internet tornou possível, pela primeira vez a comunicação de muitos para muitos, através de qualquer parte do mundo, abolindo, de vez, as barreiras do tempo e do espaço. Desde o seu aparecimento, esta tecnologia não tem parado de evoluir e todos os dias assistimos a novas possibilidades, que ela nos oferece.

A internet é uma tecnologia que veio para ficar e, são poucos os indivíduos que, atualmente não a incorporam nas suas práticas quotidianas. A sua utilização é tão natural que se pode afirmar com alguma segurança que ela é, já, uma extensão do próprio indivíduo. Em Portugal, assistiu-se a este fenómeno, sem, contudo, ignorar que existe sempre um grupo de resistentes à utilização desta tecnologia, que são, geralmente, os indivíduos mais velhos e com níveis de escolaridade mais baixa. Ainda assim, a maioria dos portugueses incorporou esta tecnologia nas suas práticas diárias, dando-lhe usos várias, onde o fator de comunicação e de procura de informação sobre temáticas várias, assumem particular relevância.

Tendo em conta este contexto, pode-se, também, aferir que é profícuo que a "internet seja abordada como o meio a privilegiar no âmbito da comuni- 
cação institucional" (Suarez et al, 2006, p. 3). As organizações encontram inúmeras vantagens de comunicação no mundo online, onde o contacto com os seus públicos é favorecido. A empresas e instituições têm, agora, um meio onde lhes é possível contruir o seu próprio discurso, sem intermediários e, onde podem comunicar com os seus consumidores em qualquer momento e a partir de qualquer lugar, de uma forma mais personalizada a interativa. " $A$ Internet é vista como um meio privilegiado de contacto entre empresas e clientes" (Marques 2012, p 29).

No conjunto de possibilidades de comunicação implementadas pela internet, concluiu-se que os websites devem ser ferramentas privilegiados na comunicação entre as organizações e os seus públicos, pelas vantagens e possibilidades que apresentam e que foram discutidas. Quando se fala de comunicação das universidades, estes meios de comunicação parecem assumir particular relevância, uma vez que se tratam de instituições onde o carácter informativo assume um papel decisivo, nas quais as páginas web se mostram como um dos primeiros meios onde os potenciais alunos ou estudantes procuram a informações desejadas.

Terminado este pequeno estudo acredita-se que os objetivos propostos foram alcançados, tendo-se encontrado resposta para a questão de partida, que era ela: porque é que os websites devem ser um meio de comunicação privilegiado no âmbito da Comunicação organizacional, em particular pelas universidades? Acredita-se, portanto, que o presente estudo representa um contributo relevante para as áreas de estudos das novas tecnologias e da comunicação organizacional.

Neste estudo apenas se discutiu uma pequena parte da importância da internet para a comunicação organizacional. Existe ainda muito terreno por desbravar neste campo de estudo. Assim, investigações futuras poderão focar-se na importância dos websites noutro tipo de empresas ou instituições ou, abordando as suas potencialidades e os desenvolvimentos que se têm registado neste meio de comunicação.

\section{REFERÊNCIAS BIBLIOGRÁFICAS}

Bargh, J. \& McKenna, K. (2004). The internet and social life. Annual Review of Psychology. 55: 573-590. 
Caraça, J. (2005). Prefácio. In G. Cardoso, A. F. Costa, C. P. Conceição \& M. C. Gomes, A Sociedade em Rede em Portugal (pp. 7-12). Porto: Campo das Letras - Editores, S.A.

Cardoso, A. (2008). A comunicação política na internet. Análise dos websites dos partidos políticos em Itália. Observatório $\left(O B S^{*}\right), 7$ : 180-204. Disponível em: http://obs.obercom.pt/index.php/obs/article/view/173/212.

Cardoso, G.; Mendonça, S.; Paisana, M. \& Lima, T. (2015). A Internet e o consumo de notícias online em Portugal 2015. Lisboa: OberCom Observatório da Comunicação.

Cardoso, G.; Mendonça, S.; Lima, T.; Paisana, M. \& Neves, M. (2014). A Internet em Portugal - sociedade em rede 2014. Lisboa: OberCom Observatório da Comunicação.

Cardoso, G.; Espanha, R.; Lima, T. \& Paisana, M. (2012). A sociedade em rede em Portugal 2012 - A internet em Portugal. Lisboa: OberCom Observatório da Comunicação.

Cardoso, G.; Firmino da Costa, A.; Palma Conceição, C. \& Carmo Gomes, M. (2005). A sociedade em rede em Portugal. Porto: Campo das Letras - Editores, S.A.

Cardoso, G.; Espanha, R. \& Gonçalves, A. (2007). A internet em Portugal (2003-2007). Lisboa: OberCom - Observatório da Comunicação.

Carrera, F. (2012). Marketing digital na versão 2.0: o que não pode ignorar. ( $2^{\mathrm{a}}$ ed). Lisboa: Edições Sílabo, Lda.

Carrillo, V. \& Ruão, T. (2005). La reputación en las universidades: de la identidad local a la reputación europea. Actas do V Congresso de Comunicação Local: 14-16, Universitat Jaume I De Castellón, Espanha.

Castells, M. (2007). A galáxia da internet reflexões sobre internet, negócios e sociedade. (R. Espanha, Trad.). (J. Oliveira \& G. Cardoso, Coord.). $\left(2^{\mathrm{a}}\right.$ ed). Lisboa: Fundação Calouste Gulbenkian. (Obra originalmente publicada em 2001).

Castells, M. (2001). A era da informação: economia, sociedade e cultura. (R. Venancio Majer \& K. Brandini Gerhardt, Trad.). (5 ed). (Vol. 1: A Sociedade em Rede). São Paulo: Editora Paz e Terra S.A. (Obra originalmente publicada em 1999). 
Comunicarte Publishing. (2010). Web trends - 10 cases made in web 2.0. Gaia: Edições Sílabo.

Direção Geral de Ensino Superior. (s.d.). Instituições de Ensino Superior Portuguesas. Disponível em: www.dges.mctes.pt/DGES/pt/Reconheciment o/NARICENIC/Ensino+Superior/Institui\%C3\%A7\%C3\%B5es+de+Ens ino+Superior+Portuguesas/

Evolução da Informática TIC - Moimenta da Beira. (s.d.). A internet em Portugal. Disponível em http://infoevo.escolasmoimenta.pt/9d06m01.

Gray, B.; Fam, K. \& Llanes, V. (2003). Branding Universities in Asian Markets. Journal of Product \& Brand Management, 2 (12): 108-120.

Jenkins, H.; Ford, S. \& Green, J. (2014) Cultura da conexão: criando valor e significado por meio da mídia negociável. (P. Arnaud, Trad.). São Paulo: Editora Aleph. (Obra originalmente publicada em 2013).

Luvizotto, C.; Fusco, E. \& Scanavacca, A. (2010). Websites educacionais: considerações acerca da arquitetura da informação no processo de ensino-aprendizagem. Educação em Revista, Marília, 11(2): 23-40.

Marques, M. (2012). Marketing e comunicação: a web como ferramenta para a promoção turística dos hotéis da Costa do Estoril. (Dissertação de Mestrado). Universidade Lusófona de Humanidades e Tecnologias, Portugal.

Marcelo, A. (2005). Novos media: inauguração de novas formas de sociabilidade. Actas do III Sopcom, VI Lusocom e II Ibérico. Covilhã: Universidade da Beira Interior.

Nunes, F. (2004). A apropriação das tecnologias de informação e comunicação na sociedade portuguesa. Scripta Nova Revista Electrónica de Geografía e Ciencias Sociales Universidad de Barcelona. 8(170): 40. Disponível em: www.ub.edu/geocrit/sn/sn-170-40.htm .

Origens da Internet. (2011). O aparecimento da internet em Portugal. Disponível em: http://otiblog1.blogspot.pt/2011/01/o-aparecimento-da-interne t-em-portugal.html.

Poupinha, L. \& Espanha, R. (2005). A existência net: o valor da net para relações públicas/ comunicação estratégica. Comunicação e Sociedade - Comunicação Estratégica, 8: 209-217. 
Póvoas, R. (2009). Relações públicas sem croquete: uma visão moderna das $R P$. Lisboa: Gestão Plus Edições.

Ruão, T.; Salgado, P.; Freitas, R. \& Ribeiro, P. (2014). Comunicação organizacional e relações públicas, numa travessia conjunta. Comunicação Organizacional e Relações Públicas: horizontes e perspetivas. Relatório de um debate, Centro de Estudos de Comunicação e Sociedade, 16-39.

Ruão, T. (2008). A comunicação organizacional e os fenómenos de identidade: a aventura comunicativa da formação da Universidade do Minho, 1974-2006. (Tese de Doutoramento em Ciências da Comunicação). Universidade do Minho, Portugal.

Ruão, T. (2005). O papel da identidade e da imagem na gestão das universidades. IV Congresso da SOPCOM - Associação Portuguesa de Ciências da Comunicação, Universidade de Aveiro, 20/21 de outubro.

Ruão, T. (2001). O conceito de identidade organizacional: teorias, gestão e valor. II Congresso da SOPCOM - Associação Portuguesa de Ciências da Comunicação, Fundação Calouste Gulbenkian, Lisboa.

Póvoas, R. (2009). Relações públicas sem croquete: uma visão moderna das $R P$. Lisboa: Gestão Plus Edições.

Suarez, A.; Moreira, R. \& Carrapatoso, E. (2006). Comunicação institucional no ensino superior: visitas virtuais e usabilidade. Conferência Ibérica de Sistemas e Tecnologias de Informação, Esposende. 